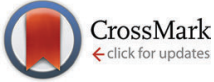

Cite this: J. Mater. Chem. B, 2016, 4, 162

Received 29th September 2015 Accepted 17th November 2015

DOI: $10.1039 / c 5 t b 02025 e$

www.rsc.org/MaterialsB

\title{
Ammonia sensing for enzymatic urea detection using organic field effect transistors and a semipermeable membrane $\dagger$
}

\author{
F. X. Werkmeister, ${ }^{a}$ T. Koide ${ }^{\text {ab }}$ and B. A. Nickel ${ }^{\text {ac }}$
}

\begin{abstract}
Organic Field Effect Transistors (OFETs) are used to measure ammonia in solution via ammonia diffusion into the OFET channel. An increase in ammonia concentrations results in a decrease in transistor currents. The regeneration of the OFET current after ammonia uptake is slow, which allows us to read out the maximum ammonia dose which was applied. A $100 \mathrm{~nm}$ parylene-C layer serves as a semipermeable top gate dielectric. The parylene layer is functionalized with the covalently attached enzyme urease. The enzyme catalyses the hydrolysis of urea to ammonia and carbon dioxide, i.e. urea can be detected via its hydrolysis product ammonia. The sensitivity covers a range of physiological concentrations of urea, which are several $\mathrm{mM}$.
\end{abstract}

\section{Introduction}

Ammonia, the end product of amino acid metabolism, is highly toxic. Therefore, ammonia is circulated only in low concentrations in body fluids, and most of it is first converted into urea in a cycle of biochemical reactions known as the urea cycle. Urea is present in body fluids in high concentrations; in healthy humans, the normal range of urea concentrations in blood is 2.5-7 mM. ${ }^{1}$ The measurement of urea concentrations is important as a marker for $e . g$. potential kidney malfunction. ${ }^{2,3}$ Specific detection of urea is possible by the enzyme urease. Urease catalyzes the hydrolysis of urea to ammonium $\left(\mathrm{NH}_{4}{ }^{+}\right)$and carbon dioxide in water:

$$
\mathrm{CO}\left(\mathrm{NH}_{2}\right)_{2}+3 \mathrm{H}_{2} \mathrm{O} \stackrel{\text { urease }}{\longrightarrow} \mathrm{CO}_{2}+2 \mathrm{NH}_{4}^{+}+2 \mathrm{OH}^{-}
$$

The $\mathrm{OH}^{-}$production of the reaction shifts the $\mathrm{pH}$ value, which has been used to detect urea using silicon transistors. ${ }^{4}$ Transistors are especially feasible for the label free measurement of target molecules, because transistors measure and at the same time amplify the signal. ${ }^{5,6}$ Organic transistors are appealing alternatives to silicon technology, since organic transistors can be mechanically flexible ${ }^{7}$ and biocompatible ${ }^{8}$ at low production cost with high throughput, e.g. with printing techniques. ${ }^{9,10}$ Furthermore, organic semiconductors can be directly functionalized with

\footnotetext{
${ }^{a}$ Fakultät für Physik \& CeNS, Ludwig-Maximilians - Universität München, München, D-80539, Germany. E-mail: nickel@lmu.de

${ }^{b}$ Japan Patent Office, 3-4-3 Kasumigaseki, Chiyoda-ku, Tokyo 100-8915, Japan

${ }^{c}$ Nanosystems Initiative Munich (NIM), D-80799 Munich, Germany

$\dagger$ Electronic supplementary information (ESI) available: Evaluation of parylene-C as top-gate dielectric. Urease activity evaluation. OFET current regeneration after exposure to ammonia. See DOI: 10.1039/c5tb02025e
}

e.g. biotin ${ }^{11}$ and interfaced with tissue. ${ }^{12}$ Organic transistors operate as sensors in liquids by two different main principles. In Organic Electrochemical Transistors (OECTs), ions diffuse into the semiconducting film and dope or de-dope the transistor channel. ${ }^{13}$ In Organic Field Effect Transistors (OFETs), the charge carrier concentration in the transistor channel is manipulated via capacitive coupling, ${ }^{14-16}$ resulting in a change of the current through the device. The source drain current $I_{\mathrm{SD}}$ of the transistor in saturation mode is given by: ${ }^{16}$

$$
I_{\mathrm{SD}}=\frac{W}{2 L} \times C \times \mu \times\left(V_{\text {gate }}-V_{\mathrm{T}}\right)^{2},
$$

with $W$ being the channel width, $L$ the channel length, $C$ the dielectric's areal capacitance, $\mu$ the mobility of the semiconductor, $V_{\text {gate }}$ the applied gate voltage and $V_{\mathrm{T}}$ the threshold voltage. Three parameters can change upon interaction with a substance of interest. First, the threshold voltage $V_{\mathrm{T}}$ may change due to electrostatic field changes, e.g. due to different $\mathrm{pH}$ values or adsorption of charged molecules. ${ }^{17}$ Second, upon binding of a substance, the capacity $C$ of the dielectric can change. ${ }^{16}$ Finally, mobility $\mu$ can change e.g. due to morphological interface effects. ${ }^{18}$ In practice, often a combination of these effects occurs. So far, organic transistors have been demonstrated for the measurement of proteins, ${ }^{9}$ glucose,${ }^{13,19}$ adrenaline, ${ }^{20}$ glutamate ${ }^{21}$ and specific ions. ${ }^{22-24}$

For enzymatic urea detection via its hydrolysis product ammonium and $\mathrm{OH}^{-}$(eqn (1)), one could consider a pH based or ion specific detection scheme. ${ }^{4,23}$ Here, a shift of the electrochemical potential due to urea hydrolysis is picked up via a shift in the electrostatic gate potential, which controls the semiconducting channel. ${ }^{4}$ This approach is limited for a weak base as 
ammonium since ammonium forms in solution a chemical equilibrium between $\mathrm{NH}_{4}{ }^{+}$and ammonia $\left(\mathrm{NH}_{3}\right)$ :

$$
\mathrm{NH}_{4}^{+}+\mathrm{H}_{2} \mathrm{O} \leftrightarrow \mathrm{NH}_{3}+\mathrm{H}_{3} \mathrm{O}^{+}
$$

Alternatively, $\mathrm{NH}_{3}$ is well known to reduce charge transport in organic semiconductors, ${ }^{25}$ most likely via creation of traps. These traps have been discussed to be caused by the interaction of the holes with the lone electron pair of $\mathrm{NH}_{3}$, as well as by dipole interaction. ${ }^{26} \mathrm{~A}$ range of ammonia gas sensors were demonstrated based on this principle. ${ }^{26-29}$ Other nitrogenous molecules like $\mathrm{NO}$ and $\mathrm{NO}_{2}$, which are biologically relevant, have also been detected in gas. ${ }^{30}$ Furthermore, it has been possible to construct arrays, which can differentiate between different vapors by the characteristic response on the organic transistors. ${ }^{31-33}$ This gas detection principle works also for organic semiconductors in direct contact with solution, as demonstrated for some molecules, e.g. cysteine ${ }^{34}$ and melamine. ${ }^{35}$

Note that another reaction product of the hydrolysis (eqn (1)) is $\mathrm{CO}_{2} . \mathrm{CO}_{2}$, however, is known to have only negligible interactions with organic transistors, ${ }^{36}$ and thus not expected to influence device characteristics. It can form a chemical equilibrium with carbonic acid and thus contribute to buffering in solution.

Here, we explore OFETs for the detection of urea. For this purpose, we fabricated a $100 \mathrm{~nm}$ parylene-C membrane onto the OFETs and functionalized the parylene-C surface with a covalently attached enzyme, urease. We suggest that urea can be detected via its hydrolysis product ammonia. Ammonia should be able to diffuse through the parylene-C membrane and give rise to a response via trapping of charge carriers in the organic semiconductor film. To determine the mechanism of sensing, i.e. $\mathrm{pH}$ vs. trapping, the response of the OFETs towards shifts of the $\mathrm{pH}$ value as well as $\mathrm{NH}_{3}$ concentration in solution is tested, with a detailed investigation of the latter. Finally, the urea concentration range, which can be detected, is determined.

\section{Experimental}

\section{OFET fabrication}

Glass slides (R. Langenbrinck) were cleaned in an ultrasonic bath with acetone, 2-propanol, and Milli-Q water for $10 \mathrm{~min}$ each. Next nominally $1.7 \mu \mathrm{m}$ parylene-C (Plasma Parylene Systems $\mathrm{GmbH}$ ) was deposited in a homebuilt CVD chamber via the Gorham route. Source and drain electrodes were defined from $20 \mathrm{~nm}$ of Au using a Lift-Off process: a bilayer of LOR 3B and S1813 G2 was spin coated, illuminated and developed using Microposit 351 Developer. The LOR 3B layer beneath the photoresist gives rise to an undercut in the dual layer during development and avoids fencing at the rims of the patterned metal electrodes after Lift-Off. Lift-Off was performed with 1165 Remover. The electric leads defined on the surface were encapsulated with a layer of SU 8 (Microchem), which was processed as recommended by the fabricant; mr-Dev 600 (Microchemicals $\mathrm{GmbH}$ ) was used as a developer. $18 \mathrm{~nm}$ DNTT (Sigma 767638, purified by one sublimation run by CreaPhys $\mathrm{GmbH}$ ) was deposited thermally onto the transistor areas through a shadow mask at a rate of $0.2 \AA^{-1}$ sith the substrates at room temperature and a base pressure of $<10^{-6}$ mbar. The devices were encapsulated by depositing $c a .100 \mathrm{~nm}$ parylene-C onto the transistor area. The area of parylene-C deposition was defined by a PDMS mask put onto the devices.

\section{Surface treatment and urease attachment}

The OFETs were treated with oxygen plasma in a plasma cleaner (50 W power, 2 mbar, $18 \mathrm{~s}$ ). Immediately afterwards, the OFETs were put into a $1 \mathrm{vol} \%$ aqueous solution of APTES (Sigma A3648) for at least $4 \mathrm{~h}$. Subsequently, the OFETs were dried under gentle nitrogen flow and put into a $5 \%$ solution of glutaraldehyde (Sigma G6257) for at least 4 hours. After drying under nitrogen flow again, the urease was bound to the surface by putting the OFETs into a solution of urease (Sigma U1500, $75 \mathrm{mg}$ ) in PBS buffer (10 mM, $15 \mathrm{ml})$.

\section{Assembly into the flow chamber}

Commercially available sticky slides IV 0.4 (Ibidi GmbH) were modified as follows: the adhesive tape was peeled off from the flow chamber and the holes necessary for the electrical contacts were drilled utilizing a CNC milling machine. After drilling the holes $467 \mathrm{MP}$ adhesive tape ( $3 \mathrm{M}$ ) was applied to the bottom of the slides and patterned according to the holes and the channel. A PtIr (Pt80/Ir20, GoodFellow, PT045110) wire was introduced into each channel after being glowed out using a Bunsen burner to serve as the electrode in the electrolyte. Finally, the OFET was attached to the modified sticky slides and the glue was given one night to obtain a good seal.

\section{Electrical measurements}

Measurements were performed using a linked system of a Keithley 2612 and 2602 source measurement unit. The source and drain contacts were hot switched using a Keithley 7072 switching matrix card. The equipment was controlled using custom written LabView programs. The potential in the electrolyte (top gate) was swept down to $-0.6 \mathrm{~V}$ versus the source contact. The source drain bias was $-0.3 \mathrm{~V}$ versus the source. All measurements were performed in ambient at $20-21{ }^{\circ} \mathrm{C}$.

\section{pH measurements in the electrolyte}

The solution for any measurement was based on $10 \mathrm{mM}$ Dulbecco's phosphate buffered saline solution without $\mathrm{Ca} / \mathrm{Mg}$ (Biochrom L 182-50, powder dissolved in Milli-Q water). Urea (Sigma U0631) and ammonia (Carl Roth 5460.1) were dissolved/ pipetted into $10 \mathrm{mM}$ DPBS solution. A HANNA $213 \mathrm{pH}$ meter was used for the $\mathrm{pH}$ measurements and calibrated before each use.

\section{AFM measurements}

AFM micrographs were recorded using a Veeco Dimension 3100 AFM in tapping mode. The software Gwyddion 2.40 was used for evaluation. 
a)

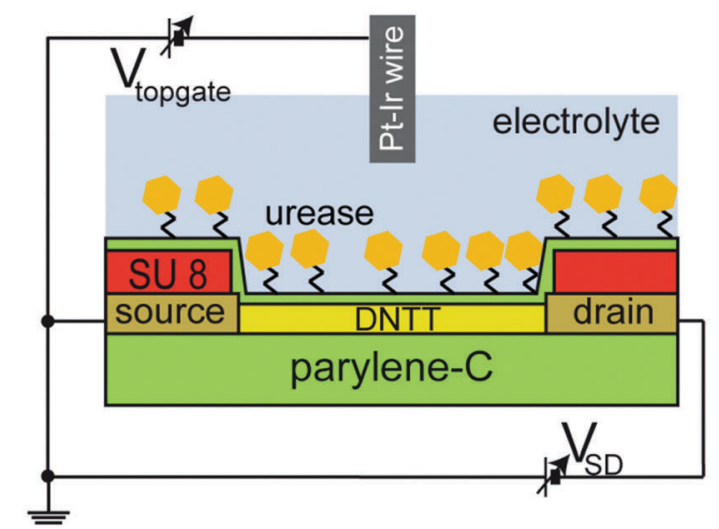

b) $0 \mu \mathrm{m}$
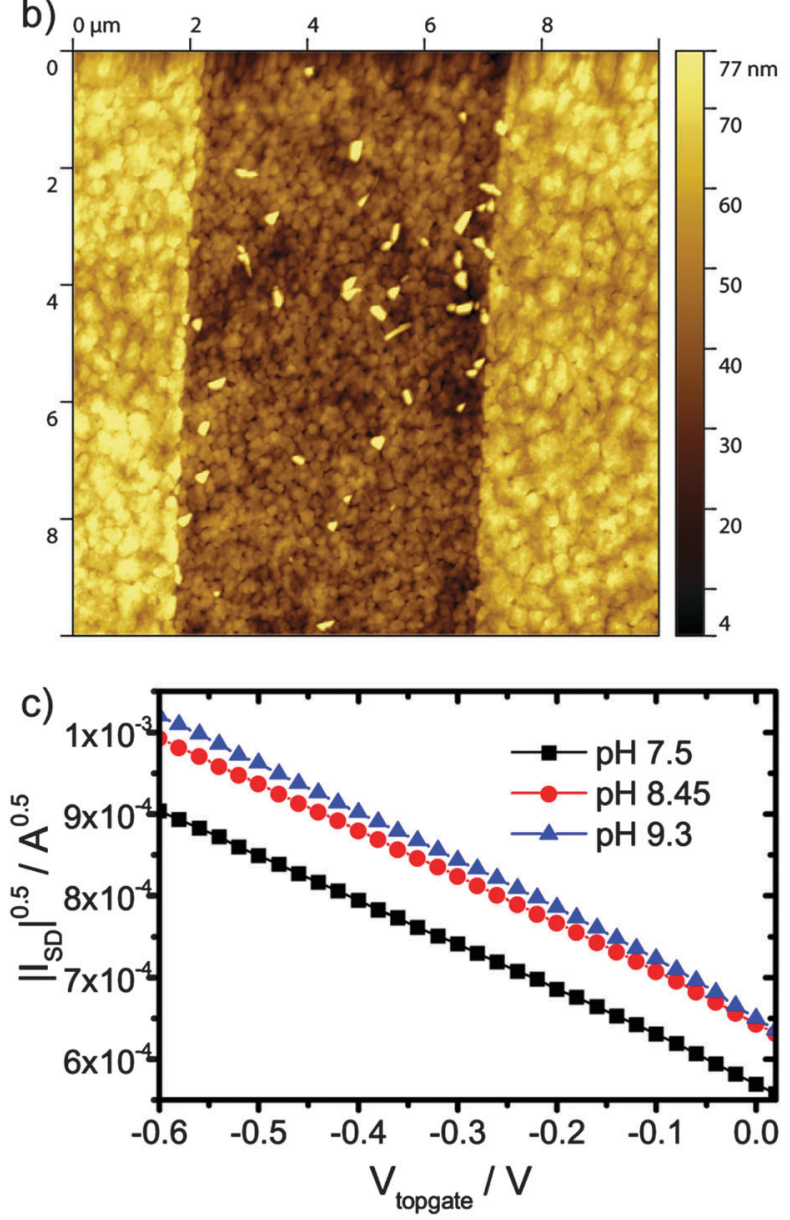

Fig. 1 (a) Schematic of the OFET device architecture and measurement scheme in solution. (b) AFM micrograph of the DNTT film in an OFET channel of length $5 \mu \mathrm{m}$. The source-drain electrodes are visible to the left and the right. (c) $\mathrm{pH}$ effect on the transconductance curve of an OFET.

\section{Results and discussion}

We designed an OFET gated via a Pt/Ir wire in solution (Fig. 1a). A parylene-C layer with source and drain electrodes patterned by photolithography for bottom contact served as a substrate. ${ }^{37}$ Dinaphtho[2,3-b:2', $\left.3^{\prime}-f\right]$ thieno[3,2-b]thiophene (DNTT) was chosen as the organic semiconductor for its stability ${ }^{38}$ and thermally deposited onto the substrate to form the organic semiconducting channel (Fig. 1b). Onto the semiconductor channel, we deposited a $100 \mathrm{~nm}$ thick parylene-C layer, which served as the top gate dielectric (ESI $\dagger$ ). Furthermore, parylene-C dielectric's surface can be functionalized by a silane coupling reaction. The functionalization procedure was based on a silane coupling reaction adapted from the functionalization of silicon dioxide dielectrics of inorganic silicon biosensors. ${ }^{4}$ To activate the parylene-C surface, it was treated with an oxygen plasma for $18 \mathrm{~s} .^{39}$ Here, the stability of DNTT versus oxidation was necessary to maintain transistor operation after the oxygen treatment. Less stable organic semiconductors might be employable, if parylene derivatives are employed, which facilitate functionalization procedures avoiding plasma activation. ${ }^{40}$ Oxygen plasma treated parylene-C surfaces were silanized by 3-aminopropyltriethoxysilane (APTES). Subsequently, glutaraldehyde and urease were bound to this surface. To verify the function of bound urease, the catalysis of urea was tested. Urea was dissolved in Milli-Q water to a concentration of $10 \mathrm{mM}$. This solution was added into a beaker together with dissolved urease or a treated parylene-C surface on glass. The $\mathrm{pH}$ value of the solution was measured against the time using a digital $\mathrm{pH}$ meter (ESI, $\dagger$ Fig. S5). The maximum value of $\mathrm{pH}$ attainable was 9.3, likely the urease degraded at this $\mathrm{pH}$ value. For the lower amount of urease, the $\mathrm{pH}$ value saturated at decreasing values. This implied a limited total number of catalyses sustainable for an individual urease enzyme. We found that the $\mathrm{pH}$ response for the bound urease was comparable to a concentration above $10 \mu \mathrm{g} \mathrm{ml}^{-1}$ of free urease in solution, albeit slower. The slower response of bound urease is likely due to mass transport from and to the surface and a larger distance of the $\mathrm{pH}$ meter from the reaction.

First, we tested the $\mathrm{pH}$ response of our OFET. The $\mathrm{pH}$ value of $10 \mathrm{mM}$ Dulbecco's phosphate buffered saline (DPBS) solution was adjusted by $\mathrm{HCl}$ and $\mathrm{NaOH}$. Increasing the $\mathrm{pH}$ value from 7.5 to 8.5 results in a positive threshold voltage shift (Fig. 1c). This shift exceeds the Nernstian limit for a single type of charged surface group. ${ }^{41}$ Most likely, the enzyme layer is responsible for this behavior. A further increase of the $\mathrm{pH}$ values from 8.45 to 9.3 and above results in small threshold voltage shifts only. Likely, the enzyme degraded (see S5, ESI $\dagger$ ) and the functionalization layer covering the transistor changed its properties. Similarly, the small increase in transconductance observed with increasing $\mathrm{pH}$ value may result from conformational changes of the urease layer. Consequently, we conclude that the bound enzyme layer is responsible for the observed $\mathrm{pH}$ response, which gives rise to an overall increase of transistor current with increasing $\mathrm{pH}$.

Second, we tested the response and sensitivity of our OFET towards ammonia in solution. Ammonia was dissolved in DPBS solution with concentrations of $0.1 \mathrm{mM}, 1 \mathrm{mM}$ and $10 \mathrm{mM}$. For $0.1 \mathrm{mM}$ solution, no change was observed, see Fig. 2a, red circles and black squares, while for a $1 \mathrm{mM}$ solution, a small change was observed (Fig. 2a, blue triangles). For an ammonia concentration of $10 \mathrm{mM}$, the transistor current reduced to half of the initial value in a matter of tens of seconds (Fig. 2b). 


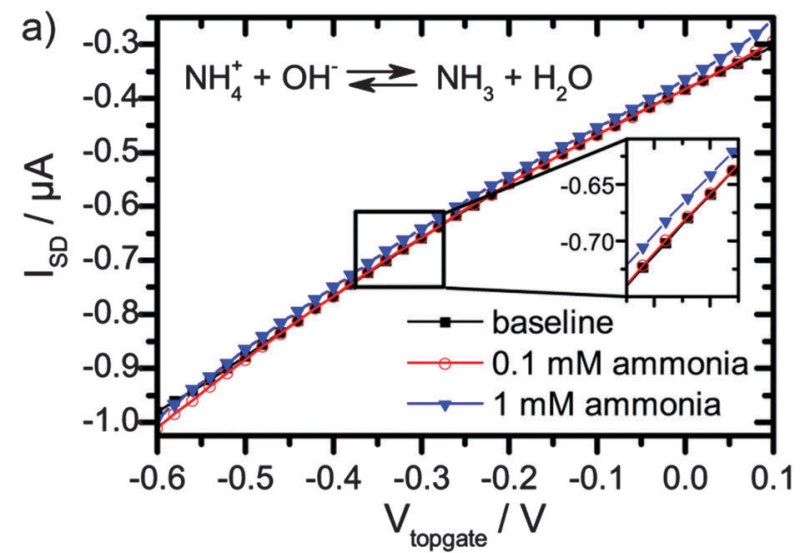

b)

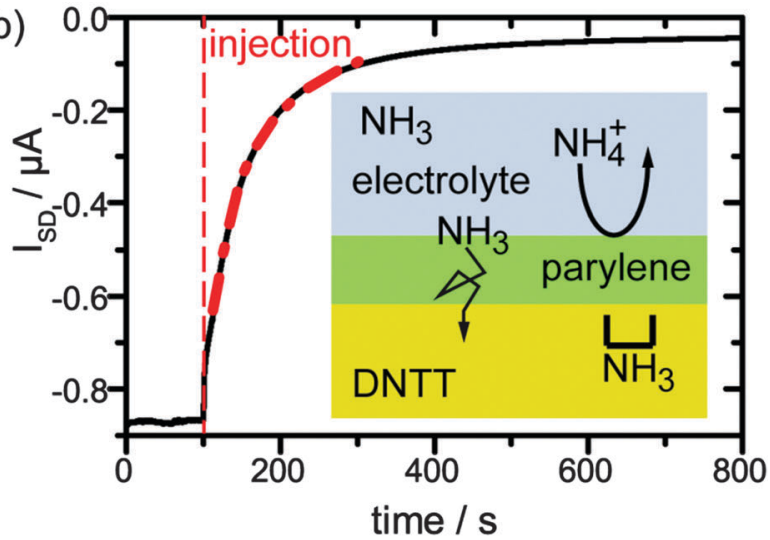

Fig. 2 (a) Effect of small ammonia concentrations $(0 \mathrm{mM}, 0.1 \mathrm{mM}$, and $1 \mathrm{mM})$ in $10 \mathrm{mM}$ DPBS buffer on the transconductance, shown as black, red, and blue curves, respectively. (b) Time resolved OFET current upon addition of $10 \mathrm{mM}$ ammonia solution with $V_{\text {topgate }}=-0.4 \mathrm{~V}$. The scheme shows the proposed interaction mechanism of ammonia with the organic semiconductor. All data were recorded at $V_{\mathrm{SD}}=-0.3 \mathrm{~V}$.

This is encouraging, because the ammonia concentrations that cause this strong response are comparable to the relevant urea concentrations in body fluids of 2.5 to $7 \mathrm{mM}^{1}$

Remarkably, the reduction of current with increasing ammonia and $\mathrm{pH}$ is opposite to the pure $\mathrm{pH}$ effect. This suggests that the current reduction dominated from $\mathrm{NH}_{3}$ passing the encapsulation layer and diffusing into the semiconductor. In order to quantify this argument, we estimate the amount of $\mathrm{NH}_{3}$ for a $1 \mathrm{mM}$ ammonia solution at the $\mathrm{pH}$ of 7.75 and for a $10 \mathrm{mM}$ solution at a pH of 10.17. The ratio of $\mathrm{NH}_{3}$ and $\mathrm{NH}_{4}{ }^{+}$depending on the $\mathrm{pH}$ is given by the Henderson-Hasselbach equation:

$$
\frac{c\left(\mathrm{NH}_{3}\right)}{c\left(\mathrm{NH}_{4}^{+}\right)}=10^{\mathrm{pH}-\mathrm{p} K_{\mathrm{a}}}
$$

here, $\mathrm{p} K_{\mathrm{a}}$ is the acid dissociation constant of $\mathrm{NH}_{4}{ }^{+42}$ The interplay between $\mathrm{pH}$ and the ratio of $\mathrm{NH}_{3}$ and $\mathrm{NH}_{4}{ }^{+}$can be found in Fig. 3; there is a rather sharp transition at $\mathrm{pH}$ values of $\sim 8$. Below $\mathrm{pH} 8$, there is an almost linear relationship between $\mathrm{NH}_{4}{ }^{+}$and $\mathrm{pH}$, i.e. one could use the $\mathrm{pH}$ value to determine the $\mathrm{NH}_{4}{ }^{+}$concentration, which in this case is also similar to the total concentration $\left(\mathrm{NH}_{4}{ }^{+}\right.$and $\left.\mathrm{NH}_{3}\right)$. However, beyond this $\mathrm{pH}$, i.e. at $\mathrm{NH}_{4}{ }^{+}$concentration beyond $2 \mathrm{mM}$, a

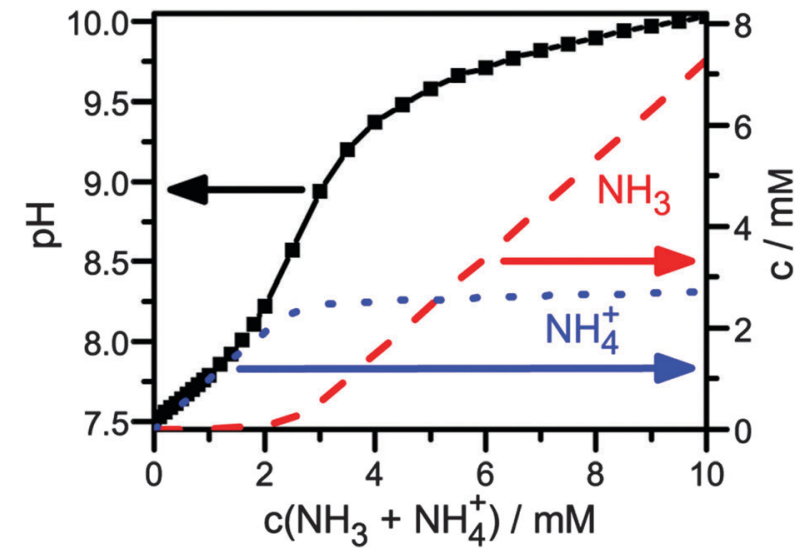

Fig. $3 \mathrm{pH}$ value and ratio of $\mathrm{NH}_{3}$ vs. $\mathrm{NH}_{4}{ }^{+}$depending on ammonia concentration in $10 \mathrm{mM}$ DPBS. The ratio was calculated assuming a $K_{a}$ of ammonia of 9.6 , since the literature value of $9.4^{42}$ for ammonia in water leads to unphysical results.

pronounced increase of $\mathrm{NH}_{3}$ concentration occurs, while the $\mathrm{NH}_{4}{ }^{+}$concentration saturates (Fig. 3). Thus, the $\mathrm{NH}_{3}$ concentration is a better indicator at high concentrations, while $\mathrm{pH}$ or $\mathrm{NH}_{4}{ }^{+}$is better suited at low concentrations.

We find that the $\mathrm{NH}_{3}$ concentration is increased by a factor of $c a .1000$ between ammonia concentrations of 1 and $10 \mathrm{mM}$, which suggest that indeed $\mathrm{NH}_{3}$ causes the transistor current drop. In fact, detection of ammonia in solution, as demonstrated here, is interesting itself, because it is an indicator of a range of diseases. ${ }^{43}$

The device response towards an increase of ammonia concentration occurs within seconds (Fig. 2b). Since our analysis suggested that the diffusion of $\mathrm{NH}_{3}$ into the semiconductor layer dominates the response, we assume that the $\mathrm{NH}_{3}$ profile is described by a 1D solution of Fick's 2 nd law of diffusion: ${ }^{19}$

$$
n(x, t)=n_{0} \operatorname{erfc}\left(\frac{x}{2 \sqrt{D t}}\right)
$$

here, $n(x, t)$ is the concentration of the diffusing species at time $t$ in distance $x$ from the reservoir with constant concentration $n_{0}$ in a material with diffusion constant $D$. We modeled the decrease of the current with this equation excluding the initial response in the first few seconds, since it includes effects from mixing. ${ }^{44}$ The fit to the data was reproducing the experimental curve well (Fig. 2b), with an extracted diffusion constant $D=$ $1.4 \times 10^{-12} \mathrm{~cm}^{2} \mathrm{~s}^{-1}$. This diffusion constant is three orders of magnitude lower than the diffusion constants for molecular oxygen and nitrogen in parylene, ${ }^{45}$ which should be similar to the one for $\mathrm{NH}_{3}$. On the other hand, the response time corresponds well to those of bare pentacene films sensing ammonia gas. $^{28,29}$ This suggests that the organic semiconductor film, which is poly-crystalline (Fig. 1b), and not the parylene layer, is the dominant diffusion barrier. Therefore, improvements on response time are more likely to be achievable by thinning or patterning engineering the organic semiconducting layer. ${ }^{26}$

While parylene is apparently no diffusion barrier for neutral $\mathrm{NH}_{3}$, it is very efficient in suppressing diffusion of charged ions $\left(\mathrm{Na}^{+}\right.$and $\left.\mathrm{Cl}^{-}\right)$to the channel region. This is because the movement 
of an ion (with radius $a$ and valency $Z$ ) from water into an nonpolar medium with dielectric constant $\varepsilon$ is prohibited by a high energy barrier, the Born charging energy $(Z e)^{2} / 8 \pi \varepsilon_{0} \varepsilon a{ }^{46}$ This effect gives rise to the high resistance of lipid bilayers. ${ }^{47}$ This principle also applies to the case of an nonpolar organic semiconductor $(\varepsilon \approx 80$ for water $^{46}$ and $\varepsilon \approx 3$ for pentacene ${ }^{14}$ ) or nonpolar encapsulation layers such as parylene. In practice, defects, e.g. pores, in nonpolar layers can lower the energy barrier, ${ }^{46}$ thus materials and processing have to be carefully chosen. ${ }^{10,48}$ In summary, the parylene acts here as a semipermeable membrane which blocks ions, while charge neutral $\mathrm{NH}_{3}$ molecules pass through rapidly. Diffusion speed in parylene decreases with the molecule size ${ }^{49}$ therefore larger molecules will also be blocked.

Upon rinsing with fresh DPBS buffer after application of $10 \mathrm{mM}$ ammonia solution, the current of the transistor recovers slowly (ESI, $\dagger$ Fig. S6). Apparently, the process of loading $\mathrm{NH}_{3}$ into the semiconducting layer was much faster than the unloading process. This is reasonable, since the $\mathrm{NH}_{3}$ gradient driving the diffusion from $10 \mathrm{mM}$ ammonia solution to the pristine semiconducting film is much steeper than the one from the $\mathrm{NH}_{3}$ loaded semiconducting film to the bare DPBS solution. Additionally, during exposure to the ammonia solution, ammonia may pass the semiconducting layer and diffuse into the parylene layer beneath the organic semiconductor. Subsequent release would take place via diffusion through the organic semiconductor with a very low rate, i.e. the parylene layer beneath the organic semiconductor acts as a reservoir.

Finally, the urea sensing of OFETs with bound urease was evaluated. Different concentrations of DPBS solutions with urea were prepared and pipetted into the flow channel of the sensor after completion of the first gate sweep. Gate sweeps were recorded continuously and the change of the transconductance curve after fixed time ( $5 \mathrm{~min}$ ) was evaluated (Fig. 4 , red data points). The first

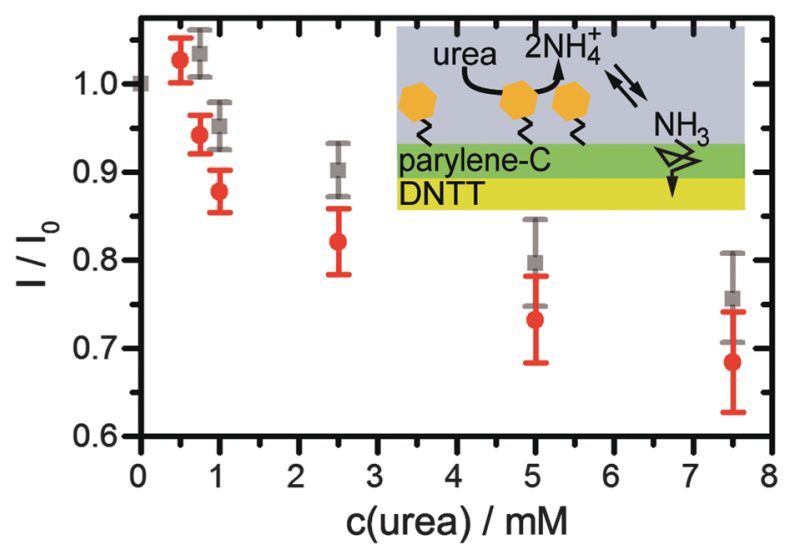

Fig. 4 Influence of increasing urea concentrations on transistor current. The first source-drain current $I_{\mathrm{SD}}$ at $V_{\mathrm{SD}}=-0.3 \mathrm{~V}$ and $V_{\text {topgate }}=-0.3 \mathrm{~V}$ (without urea) is used as reference current $I_{0}$ (grey data point). The resultant ratio $I_{\mathrm{SD}} / I_{0}$ (red dots) is displayed as recorded after 5 min exposure to a given urea concentration. After each exposure, and prior to the next exposure the OFETs are rinsed with fresh buffer by exchanging the liquid volume 5 times. The ratio $I_{\mathrm{SD}} / I_{0}$ prior to each urea exposure is shown after rinsing with buffer as grey squares. The data points are an average of the data of 3 different OFETs with the error bars giving the standard deviation. response was detected for a urea concentration of $0.75 \mathrm{mM}$. Between the different urea concentrations, we rinsed the flow channel with fresh DPBS buffer by replacing the complete liquid volume 5 times with fresh buffer. Due to the slow recovery, the transistor current stayed at the low level (Fig. 4, gray data points) suggesting that the device is best suited to monitor the maximal dose that the semiconductor film has seen. We observed a systematic decrease of the current up to a urea concentration of $7.5 \mathrm{mM}$, i.e. the device covers the full urea range in a healthy patient. In comparing the response in Fig. 4 to the ammonia concentration dependence in Fig. 3 one may note that the response starts before the pronounced increase in $\mathrm{NH}_{3}$ in Fig. 3. Already below the pronounced increase in $\mathrm{NH}_{3}$ at $2 \mathrm{mM}$ in Fig. 3 the $\mathrm{NH}_{3}$ concentration is nonzero and linearly increasing. Organic transistors are sensitive to small amounts of $\mathrm{NH}_{3},{ }^{29,50}$ as also observed here in the detection of $1 \mathrm{mM}$ ammonia (Fig. 2a). Furthermore, the response of organic transistors with increasing $\mathrm{NH}_{3}$ tends to saturate, ${ }^{50}$ which is also seen here for higher urea/ ammonia concentrations (Fig. 4). After some measurement cycles, our OFETs did not react any more to the addition of urea, even after long times of regeneration. This is not unexpected, since urease was expected to sustain only a limited number of reactions before degradation. Hence, the device is best used as a disposable (use once) sensor, very much in agreement with what is needed in typical healthcare applications.

Now, we compare the sensitivity of our device with other detection schemes. In electrochemical detection, the reaction is coupled to a redox species and the corresponding charge transfer is recorded. This way a detection range of $0.8-16.6 \mathrm{mM}$ could be realized. ${ }^{51}$ Another transduction mechanism is to record the potential shift of a $\mathrm{pH}$ sensitive device due to the change of the $\mathrm{pH}$ value by the hydrolysis of urea in solution. ${ }^{4}$ A detection range of 0.05-10 $\mathrm{mM}$ was reported for urease functionalized polymer membranes coupled to an external FET. ${ }^{52}$ Both schemes used $\mathrm{Ag} / \mathrm{AgCl}$ reference electrodes, which are hard to miniaturize ${ }^{53}$ and integrate into fabrication schemes. Here, we propose a detection scheme, which covers the urea range of medical samples. Our detection scheme relies on $\mathrm{NH}_{3}$ concentration. Since the $\mathrm{NH}_{3}$ interaction dominates electrochemical effects, it is less affected by potential changes. This makes it possible to use a simple Pt/Ir wire as a gate electrode in the electrolyte. A Pt electrode $^{13}$ can be included as a planar electrode on top of the device during fabrication of the OFET.

\section{Conclusions}

In conclusion, we demonstrated an OFET configuration, which allows the measurement of $\mathrm{NH}_{3}$ in aqueous solution. The OFET shows a rapid current decrease within seconds due to the diffusion of $\mathrm{NH}_{3}$ through a semipermeable parylene membrane into the organic semiconducting layer. Therefore, in applications where organic electronic devices are to work in a biological environment, undesired influences of $\mathrm{NH}_{3}$ should be taken into account. Furthermore, such OFETs can detect physiologically relevant urea concentrations of $0.75 \mathrm{mM}$ to $7.5 \mathrm{mM}$ due to urea 
hydrolysis into $\mathrm{NH}_{3}$ by anchoring urease to the membrane. Regeneration of transistor current is slow, thus the device is well suited for the readout of the maximal urea dose that the device has been exposed to. In turn, the readout may be performed after exposure to e.g. body fluids, which may be interesting for diagnosis purposes. We expect that OFETs with thin and micro-/nanopatterned organic semiconductor layers will show improved sensitivity as well as faster response and recovery times. This case study shows that gas sensing of partial pressure ${ }^{32}$ and sensing of molar concentration in solution are similar. In liquid, a non-polar semipermeable membrane allows us to separate neutral molecules from the charged species and stabilizes the device. This opens up interesting avenues for design of biosensors utilizing organic semiconductors.

\section{Acknowledgements}

We would like to thank Prof. Kersting (LMU \& CeNS) for providing access to the parylene coater. We acknowledge funding from the Deutsche Forschungsgemeinschaft (DFG) through the Sonderforschungsbereich SFB 1032 "Nanoagents" project A07. Teru Koide would like to thank the Japan patent office for the opportunity of his research stay at the LMU.

\section{References}

1 I. Gotsman, D. Zwas, D. Planer, D. Admon, C. Lotan and A. Keren, Medicine, 2010, 89, 197-203.

2 M. Singh, N. Verma, A. K. Garg and N. Redhu, Sens. Actuators, B, 2008, 134, 345-351.

3 G. Dhawan, G. Sumana and B. D. Malhotra, Biochem. Eng. J., 2009, 44, 42-52.

4 D. Niwa, K. Omichi, N. Motohashi, T. Homma and T. Osaka, Sens. Actuators, B, 2005, 108, 721-726.

5 A. Poghossian and M. J. Schöning, Electroanalysis, 2014, 26, 1197-1213.

6 D. Khodagholy, J. Rivnay, M. Sessolo, M. Gurfinkel, P. Leleux, L. H. Jimison, E. Stavrinidou, T. Herve, S. Sanaur, R. M. Owens and G. G. Malliaras, Nat. Commun., 2013, 4, 2133.

7 P. Leleux, C. Johnson, X. Strakosas, J. Rivnay, T. Hervé, R. M. Owens and G. G. Malliaras, Adv. Healthcare Mater., 2014, 3, 1377-1380.

8 G. Scarpa, A. L. Idzko, S. Götz and S. Thalhammer, Macromol. Biosci., 2010, 10, 378-383.

9 M. L. Hammock, O. Knopfmacher, T. N. Ng, J. B. H. Tok and Z. Bao, Adv. Mater., 2014, 26, 6138-6144.

10 M. Medina-Sánchez, C. Martínez-Domingo, E. Ramon and A. Merkoçi, Adv. Funct. Mater., 2014, 24, 6291-6302.

11 E. D. Glowacki, R. R. Tangorra, H. Coskun, D. Farka, A. Operamolla, Y. Kanbur, F. Milano, L. Giotta, G. M. Farinola and N. S. Sariciftci, J. Mater. Chem. C, 2015, 3, 6554-6564.

12 S. Loffler, B. Libberton and A. Richter-Dahlfors, J. Mater. Chem. B, 2015, 3, 4979-4992.
13 D. A. Bernards, D. J. Macaya, M. Nikolou, J. A. DeFranco, S. Takamatsu and G. G. Malliaras, J. Mater. Chem., 2008, 18, 116-120.

14 M. Göllner, G. Glasbrenner and B. Nickel, Electroanalysis, 2012, 24, 214-218.

15 F. Buth, D. Kumar, M. Stutzmann and J. A. Garrido, Appl. Phys. Lett., 2011, 98, 153302.

16 G. Palazzo, D. De Tullio, M. Magliulo, A. Mallardi, F. Intranuovo, M. Y. Mulla, P. Favia, I. Vikholm-Lundin and L. Torsi, Adv. Mater., 2014, 27, 911-916.

17 M. Demelas, S. Lai, A. Spanu, S. Martinoia, P. Cosseddu, M. Barbaro and A. Bonfiglio, J. Mater. Chem. B, 2013, 1, 3811-3819.

18 M. Magliulo, K. Manoli, E. Macchia, G. Palazzo and L. Torsi, Adv. Mater., 2014, DOI: 10.1002/adma.201403477.

19 D. Elkington, W. J. Belcher, P. C. Dastoor and X. J. Zhou, Appl. Phys. Lett., 2014, 105, 043303.

20 N. Coppede, G. Tarabella, M. Villani, D. Calestani, S. Iannotta and A. Zappettini, J. Mater. Chem. B, 2014, 2, 5620-5626.

21 L. Kergoat, B. Piro, D. T. Simon, M.-C. Pham, V. Noël and M. Berggren, Adv. Mater., 2014, 26, 5658-5664.

22 M. Sessolo, J. Rivnay, E. Bandiello, G. G. Malliaras and H. J. Bolink, Adv. Mater., 2014, 26, 4803-4807.

23 K. Melzer, A. M. Münzer, E. Jaworska, K. Maksymiuk, A. Michalska and G. Scarpa, Org. Electron., 2014, 15, 595-601.

24 T. Minami, T. Minamiki and S. Tokito, Chem. Commun., 2015, 51, 9491-9494.

25 A. Assadi, G. Gustafsson, M. Willander, C. Svensson and O. Inganäs, Synth. Met., 1990, 37, 123-130.

26 L. Li, P. Gao, M. Baumgarten, K. Müllen, N. Lu, H. Fuchs and L. Chi, Adv. Mater., 2013, 25, 3419-3425.

27 J. W. Jeong, Y. D. Lee, Y. M. Kim, Y. W. Park, J. H. Choi, T. H. Park, C. D. Soo, S. M. Won, I. K. Han and B. K. Ju, Sens. Actuators, B, 2010, 146, 40-45.

28 J. S. Yu, X. G. Yu, L. Zhang and H. J. Zeng, Sens. Actuators, B, 2012, 173, 133-138.

29 X. Yu, N. Zhou, S. Han, H. Lin, D. B. Buchholz, J. Yu, R. P. H. Chang, T. J. Marks and A. Facchetti, J. Mater. Chem. C, 2013, 1, 6532-6535.

30 F. Marinelli, A. Dell'Aquila, L. Torsi, J. Tey, G. P. Suranna, P. Mastrorilli, G. Romanazzi, C. F. Nobile, S. G. Mhaisalkar, N. Cioffi and F. Palmisano, Sens. Actuators, B, 2009, 140, 445-450.

31 L. Torsi, A. Dodabalapur, L. Sabbatini and P. G. Zambonin, Sens. Actuators, B, 2000, 67, 312-316.

32 B. Crone, A. Dodabalapur, A. Gelperin, L. Torsi, H. E. Katz, A. J. Lovinger and Z. Bao, Appl. Phys. Lett., 2001, 78, 2229.

33 W. Huang, J. Sinha, M.-L. Yeh, J. F. M. Hardigree, R. LeCover, K. Besar, A. M. Rule, P. N. Breysse and H. E. Katz, Adv. Funct. Mater., 2013, 23, 4094-4104.

34 M. E. Roberts, S. C. Mannsfeld, N. Queralto, C. Reese, J. Locklin, W. Knoll and Z. Bao, Proc. Natl. Acad. Sci. U. S. A., 2008, 105, 12134-12139. 
35 H. Chen, S. Dong, M. Bai, N. Cheng, H. Wang, M. Li, H. Du, S. Hu, Y. Yang, T. Yang, F. Zhang, L. Gu, S. Meng, S. Hou and X. Guo, Adv. Mater., 2015, 27, 2113-2120.

36 M. Mirza, J. Wang, L. Wang, J. He and C. Jiang, Org. Electron., 2015, 24, 96-100.

37 F. Werkmeister and B. Nickel, J. Mater. Chem. B, 2013, 1, 3830-3835.

38 T. Yamamoto and K. Takimiya, J. Am. Chem. Soc., 2007, 129, 2224-2225.

39 K. Länge, S. Grimm and M. Rapp, Sens. Actuators, B, 2007, 125, 441-446.

40 C.-H. Chang, S.-Y. Yeh, B.-H. Lee, C.-W. Hsu, Y.-C. Chen, C.-J. Chen, T.-J. Lin, M. Hung-Chih Chen, C.-T. Huang and H.-Y. Chen, J. Mater. Chem. B, 2014, 2, 8496-8503.

41 P. Bergveld, ISFET, Theory and Practice, Toronto, 2003.

42 R. G. Bates and G. D. Pinching, J. Res. Natl. Bur. Stand., 1949, 42, 419-430.

43 T. A. Kumar, E. Capua, M. Tkachev, S. N. Adler and R. Naaman, Adv. Funct. Mater., 2014, 24, 5833-5840.
44 M. L. Hammock, O. Knopfmacher, B. D. Naab, J. B. H. Tok and Z. Bao, ACS Nano, 2013, 7, 3970-3980.

45 Y. S. Yeh, W. J. James and H. Yasuda, J. Polym. Sci., Part B: Polym. Phys., 1990, 28, 545-568.

46 A. Parsegian, Nature, 1969, 221, 844-846.

47 S. Gritsch, P. Nollert, F. Jahnig and E. Sackmann, Langmuir, 1998, 14, 3118-3125.

48 M. Göllner, M. Huth and B. Nickel, Adv. Mater., 2010, 22, 4350-4354.

49 S. C. George and S. Thomas, Prog. Polym. Sci., 2001, 26, 985-1017.

50 M. Mirza, J. Wang, D. Li, S. A. Arabi and C. Jiang, ACS Appl. Mater. Interfaces, 2014, 6, 5679-5684.

51 M. Tak, V. Gupta and M. Tomar, J. Mater. Chem. B, 2013, 1, 6392-6401.

52 N. C. S. Vieira, A. Figueiredo, E. G. R. Fernandes, F. E. G. Guimaraes and V. Zucolotto, Synth. Met., 2013, 175, 108-111.

53 A. W. Hassel, K. Fushimi and M. Seo, Electrochem. Commun., 1999, 1, 180-183. 